\title{
Warthin-like and classic papillary thyroid cancer have similar clinical presentation and prognosis
}

${ }^{1}$ Departamento de Endocrinología, Facultad de Medicina, Pontificia Universidad Católica de Chile, Santiago, Chile ${ }^{2}$ Centro Traslacional de Endocrinología (CETRENUC), Facultad de Medicina, Pontificia Universidad Católica de Chile, Santiago, Chile

${ }^{3}$ Departamento de Anatomía Patológica, Facultad de Medicina, Pontificia Universidad Católica de Chile, Santiago, Chile

${ }^{4}$ Departamento de Cirugía de Cabeza y Cuello, Facultad de Medicina, Pontificia Universidad Católica de Chile, Santiago, Chile ${ }^{5}$ Clínica Las Condes, Santiago, Chile
Correspondence to: José Miguel Domínguez Diagonal Paraguay, 362, piso 4, Santiago, Región Metropolitana, Chile jomingu@uc.cl

Received on Jan/12/2020 Accepted on Apr/27/2020

DOI: 10.20945/2359-3997000000270

\author{
Roberto Olmos ${ }^{1,2}$ \\ https://orcid.org/0000-0002-7129-9543 \\ Francisco Muñoz ${ }^{1}$ \\ https://orcid.org/0000-0003-4170-7056 \\ Francisca Donoso ${ }^{1}$ \\ https://orcid.org/0000-0003-2080-0956 \\ Jorge López ${ }^{1}$ \\ https://orcid.org/0000-0002-7449-7035 \\ María José Bruera ${ }^{1}$ \\ https://orcid.org/0000-0001-7921-5829 \\ Magdalena Ruiz-Esquide \\ https://orcid.org/0000-0002-6954-1265 \\ Lorena Mosso ${ }^{1,2}$ \\ https://orcid.org/0000-0001-5420-7099 \\ Nicole Lustig ${ }^{1,2}$ \\ https://orcid.org/0000-0001-8019-1175 \\ Antonieta Solar ${ }^{3}$ \\ https://orcid.org/0000-0003-0764-2068 \\ Nicolás Droppelmann 4 \\ https://orcid.org/0000-0001-9676-2613 \\ Pablo H. Montero 5 \\ https://orcid.org/0000-0002-1297-9469 \\ Hernán E. González ${ }^{4}$ \\ https://orcid.org/0000-0001-6887-3455 \\ José Miguel Domínguez ${ }^{1,2}$ \\ https://orcid.org/0000-0002-6157-8055
}

\section{ABSTRACT}

Objective: Warthin-like papillary thyroid cancer (WL-PTC) is an uncommon variant of PTC, usually associated with lymphocytic thyroiditis. Scarce evidence suggests that WL-PTC has similar clinical presentation to classic PTC (C-PTC), with no studies comparing risks of recurrence and response to treatment between both variants. Our objective was to describe the clinical presentation and prognosis of WL-PTC and compare it to C-PTC. Subjects and methods: Retrospective analysis of a prospective cohort, including 370 (96\%) patients with C-PTC and 17 (4\%) with WL-PTC, consecutively treated with total thyroidectomy with or without RAl, followed for at least 6 months. We compared clinical presentation, risk of mortality and recurrence, as well as response to treatment between both variants. Results: Of the total cohort: 317 (82\%) female, $38 \pm 13.5$ years, median follow-up 4 years (0.5-28.5); most of them stage I and low/intermediate risk of recurrence. We found no differences regarding clinical-pathological data and risk of recurrence. WL-PTC was associated with a higher rate of anti-thyroglobulin antibodies (TgAb) $(65 \%$ vs. $36 \%, p=0.016)$ and lymphocytic thyroiditis $(59 \%$ vs. $34 \%, p=0.03)$. The rates of biochemical and structural incomplete responses were similar in both variants. WL-PTC had a lower rate of excellent response $(23 \%$ vs. $54 \%, p=0.01)$, which became non-significant when performing analysis by TgAb presence (50\% vs. $67 \%, p=N S)$. Conclusions: WL-CPT and C-CPT have similar clinical presentation and rate of recurrence. The lower rate of excellent response to treatment in WL-PTC is due to a higher frequency of TgAb. WL-PCT should not be considered an aggressive variant of PTC. Arch Endocrinol Metab. 2020;64(5):542-7

\section{Keywords}

Thyroid cancer, papillary; anti-thyroglobulin antibody; Warthin-like papillary thyroid cancer 


\section{INTRODUCTION}

Papillary thyroid carcinoma (PTC) is the most common malignant endocrine neoplasia (1). The classic variant encompasses $>90 \%$ of PTC, but many other subtypes have been described according to specific histopathological hallmarks. This distinction has clinical implications because some of these variants, including tall cell, solid and columnar, have a more aggressive clinical behavior, consistently associated with less disease-free survival and cancer specific survival (2).

One of the most recently described PTC subtype is the Warthin-like variant (WL-PTC), first published in 1995 by Apel and cols., and owing its name due to its close resemblance to the papillary lymphomatous cystadenoma, or Warthin tumor of the salivary glands (3). Histologically, WL-PTC is characterized by oncocytic cells lining the papillae, with a dense lymphoplasmacytic infiltrate of the papillary stalks, usually surrounded by lymphocytic thyroiditis $(4,5)$.

While previously deemed a subtype of the oncocytic variant of PTC, the 4th edition of the World Health Organization (WHO) Classification of Tumors of Endocrine Organs classified WL-PTC as a distinct variant (5). Although increasingly described by pathologists, WL-PTC is still considered an unusual PTC variant, accounting for $0.2 \%-1 \%$ of PTC, affecting predominantly middle-aged women (6).

The available published data suggest that WLPTC has a similar clinical behavior to classic papillary thyroid cancer (C-PTC), with comparable rates of extrathyroidal extension, vascular invasion and nodal metastases (6). However, to the best of our knowledge, no study has directly compared the risks of recurrence and death, as well as the response to treatment between WL-PTC and C-PTC. Our goal was to analyze the clinical presentation and prognosis between WL-PTC and C-PTC treated in a single institution.

\section{SUBJECTS AND METHODS}

\section{Subjects}

This study was approved by the ethic committee of our institution.

Patients 18 years-old or older, consecutively treated and followed prospectively at our institution between December 2012 and December 2018, who met the following criteria were initially selected: (i) diagnosis of classic or WL-PTC; (ii) subjected to total thyroidectomy, with neck dissection performed only with evidence of clinically apparent nodal disease detected at clinical examination, preoperative neck ultrasound (US), or during intra-operative inspection by the surgeon; and (iii) followed for at least 6 months. Nodal disease was classified in low and high volume according to 2015 American Thyroid Association (ATA) guideline (7). All biopsies were reviewed by an experienced pathologist (A.S.).

The need for radioiodine (RAI) was defined in each patient following the recommendations of the 2009 ATA guidelines until December 2015, and the 2015 ATA guidelines were followed starting January $2016(7,8)$. Patients were classified according to the ATA 2015 recurrence risk category (low, intermediate and high) as well as the eighth edition of the AJCC/ UICC staging system (I, II, III and IV) based on the preoperative neck US, intra-operative findings and final surgical pathology report $(7,9)$.

Patients who met any of the following criteria were excluded: (i) partial thyroidectomy; (ii) absence of or incomplete surgical pathology report; (iii) follow-up of less than 6 months; or (iv) Tg, TgAb or neck US not performed during follow-up.

\section{Follow-up}

After initial treatment, all patients were treated with a levothyroxine (LT4) dose sufficient to maintain a serum thyrotropin $(\mathrm{TSH})<1.0 \mathrm{uUI} / \mathrm{mL}$. The patients were followed with clinical examination, $\mathrm{Tg}, \mathrm{TgAb}$ and neck US every 6 months during the first year and thereafter at 6-12-month interval according to their response to treatment, at the attending physician's discretion.

\section{Outcomes}

Cervical neck US was performed by experienced radiologists. Negative neck US was defined as either normal exploration or the presence of any of the following findings: (i) thyroid bed avascular nodules $\leq 5 \mathrm{~mm}$ in diameter, stable during follow-up, or (ii) reactive cervical lymph nodes. Disease recurrence/ persistence was defined as suspicious findings at images, confirmed with cytopathologic study and $\mathrm{Tg}$ measurement on aspirate washout.

Response to treatment was defined according to US, $\mathrm{Tg}$ and $\mathrm{TgAb}$ findings during follow-up. Excellent response was defined when all of the following were 
present: negative US, non-stimulated $\mathrm{Tg}<0.2 \mathrm{ng} / \mathrm{mL}$ and negative $\mathrm{TgAb}$. Incomplete biochemical response was defined in the presence of negative US and either non-stimulated $\mathrm{Tg}>1.0 \mathrm{ng} / \mathrm{mL}$ in RAI ablated patients or $>5.0 \mathrm{ng} / \mathrm{mL}$ in non-RAI ablated patients, plus negative $\mathrm{TgAb}$, or positive $\mathrm{TgAb}$ with an increase $\geq 50 \%$ in their concentration. Incomplete structural response was defined as highly suspicious neck US (or any other image modality), confirmed with cytological study, regardless of $\mathrm{Tg}$ or $\mathrm{TgAb}$ serum concentration. Indeterminate response was defined either as (i) negative neck US plus Tg $\geq 0.2-\leq 1.0 \mathrm{ng} / \mathrm{mL}$ in RAI ablated or $\leq 5.0 \mathrm{ng} / \mathrm{mL}$ in non RAI ablated patients, plus $\operatorname{TgAb}(-)$ or (ii) negative US plus $\operatorname{TgAb}(+)$ with decreasing or stable concentration during follow-up (increase $<50 \%$ in their concentration) or (iii) nonspecific findings on imaging studies (10-12).

\section{Assays}

Tg was measured with a chemiluminescent immunoassay with a functional sensitivity of $0.1 \mathrm{ng} / \mathrm{mL}$ (Elecsys II, Roche Diagnostics, Rotkreutz, Switzerland). TgAb was also measured with a chemiluminescent immunoassay (Architect il000, Abbot Laboratories, Abbott Park, IL), with a reference value of up to $4.11 \mathrm{IU} / \mathrm{mL}$ and an analytical sensitivity of $1.0 \mathrm{IU} / \mathrm{mL}$. Both $\mathrm{Tg}$ and $\mathrm{TgAb}$ were normalized with CRM 457.

\section{Statistical analysis}

Continuous variables are presented either as mean and standard deviation or median with range as appropriate. Categorical comparisons were performed with Fisher's exact test, and continuous variables were compared using Student's t-test. A p-value of $<0.05$ was considered significant. Statistical analysis was performed using SPSS (v.21.0.0: SPSS, Inc., Chicago, IL).

\section{RESULTS}

Of the 462 patients of this cohort, 387 met inclusion criteria for this study, and 75 were excluded due to lack of follow-up or insufficient clinical and pathological information. Of the included cohort, $317(82 \%)$ patients were female, with a mean age of $38 \pm 13.5$ years, followed for a median of $4.0(0.5-28.5)$ years. Total thyroidectomy alone was performed in 221
(57\%) patients, and total thyroidectomy with lymph node dissection in 166 (43\%) patients (Table 1).

Of the cohort, $370(96 \%)$ had C-PTC, and $17(4 \%)$ had WL-PTC. Fourteen (82\%) of 17 WL-PTC were diagnosed since 2015. As shown in table 1 , most patients had stage I PTC, and 95\% were classified as low or intermediate risk of recurrence (Table 1). No differences were found in gender, age, extension of surgery, major histopathological features, risk of recurrence and AJCC eighth classification (Table 1).

Compared with C-PTC, WL-PTC was associated with a higher rate of positive $\operatorname{TgAb}(65 \%$ vs. $36 \%, \mathrm{p}=$ $0.016)$ and lymphocytic thyroiditis $(59 \%$ vs. $34 \%, \mathrm{p}=$ $0.03)$. RAI ablation was performed in $66(35 \%)$ of WLPTC and $238(64 \%)$ of C-PTC ( $=0.017)$. During the evaluated period, there was a trend towards selective use of RAI: before 2015 it was indicated more commonly (172 of $224,(77 \%))$, in contrast to a more restrictive indication since 2015 (72 of $163(44 \%)(\mathrm{p}<0.0001)$. Since 2015, 68 of $149(45 \%)$ and 4 of $14(29 \%)$ of C-PTC and WL-PTC received RAI, respectively (p NS). The median follow-up was 2.1 (0.6-6.6) years in WL-PTC and $4.1(0.5-28.5)$ years in C-PTC patients $(\mathrm{p}=0.022)$ (Table $\mathrm{l})$.

The rates of both biochemical and structural incomplete responses were similar between WL-PTC and C-PTC (Table 2). During follow-up, 39 (10.5\%) patients had incomplete response: $14(3.8 \%)$ biochemical and $25(6.8 \%)$ structural disease. When the analysis was conducted according to the year of diagnosis, in patients treated since 2015 , during which RAI administration was similar between both variants, the rate of recurrence remained similar for C-PTC and WL-PTC (Table 2).

At the end of follow-up, WL-PTC had a lower rate of excellent response than C-PTC: 4 (23\%) vs. $202(54 \%)(\mathrm{p}=0.01)$. Interestingly, when subgroup analysis was performed according to the presence of $\operatorname{TgAb}$, this difference became non-significant. In the $\mathrm{TgAb}$ negative group, excellent response was achieved in $3(50 \%)$ C-PTC vs. 150 (67\%) patients with WL-PTC $(\mathrm{p}=0.64)$, whereas in the TgAb positive group, this outcome occurred in 1 (9.1\%) C-PTC vs. $43(34 \%)$ WL-PTC $(\mathrm{p}=0.25)$ patients (Table 3$)$.

Of all $\operatorname{TgAb}(+)$ patients $(\mathrm{n}=143)$, at the end of follow-up 44 (31\%) patients achieved excellent response and in $39(27 \%) \mathrm{TgAb}$ concentration decreased $\geq 50 \%$ with a negative neck US. 
Table 1. Clinical presentation of the 387 included patients

\begin{tabular}{|c|c|c|c|c|c|}
\hline & & & C-PTC $(n=370)$ & WL-PTC $(n=17)$ & P-value \\
\hline \multicolumn{3}{|l|}{ Age (years) } & $38 \pm 13$ & $39 \pm 16$ & NS \\
\hline \multicolumn{3}{|l|}{ Female Gender } & $301(81 \%)$ & $16(94 \%)$ & NS \\
\hline \multicolumn{3}{|c|}{ Length of follow-up (years) } & $4.1(0.5-28.5)$ & $2.0(0.6-6.6)$ & 0.022 \\
\hline \multirow[t]{2}{*}{ Type of surgery } & TT & & $212(57 \%)$ & $9(53 \%)$ & NS \\
\hline & $T T+L N D$ & & $158(43 \%)$ & $8(47 \%)$ & \\
\hline \multirow[t]{8}{*}{ Histology } & Diameter (cm) & & $1.1(0.1-7.0)$ & $1.5(0.4-3.0)$ & NS \\
\hline & Multifocality & No & $230(62 \%)$ & $7(41 \%)$ & NS \\
\hline & & Yes & $140(38 \%)$ & 10 (59\%) & NS \\
\hline & ETE & No & $253(68.3 \%)$ & $14(82.4 \%)$ & \\
\hline & & Microscopic & $105(28.4 \%)$ & $3(17.6 \%)$ & NS \\
\hline & & Macroscopic & $12(3.3 \%)$ & $0(0 \%)$ & \\
\hline & Lymphovascular invasion & & $78(21 \%)$ & $2(12 \%)$ & NS \\
\hline & Lymphocytic thyroiditis & & $125(34 \%)$ & $10(59 \%)$ & 0.03 \\
\hline \multicolumn{2}{|l|}{ Positive TgAb } & & $132(36 \%)$ & $11(65 \%)$ & 0.016 \\
\hline \multirow[t]{6}{*}{ T } & $1 \mathrm{a}$ & & $185(50 \%)$ & $4(23.5 \%)$ & NS \\
\hline & $1 b$ & & $120(32.4 \%)$ & $8(47.1 \%)$ & \\
\hline & 2 & & $43(11.6 \%)$ & $5(29.4 \%)$ & \\
\hline & 3a & & $5(1.4 \%)$ & $0(0 \%)$ & \\
\hline & $3 b$ & & $4(1.1 \%)$ & $0(0 \%)$ & \\
\hline & $4 a$ & & $13(3.5 \%)$ & $0(0 \%)$ & \\
\hline \multirow[t]{3}{*}{ N } & 0 & & 206 (55.7\%) & $10(58.8 \%)$ & NS \\
\hline & $1 \mathrm{a}$ & & 87 (23.5\%) & $3(17.6 \%)$ & \\
\hline & $1 b$ & & 77 (20.8\%) & $4(23.5 \%)$ & \\
\hline \multirow[t]{2}{*}{ M } & 0 & & 365 (98.6\%) & $17(100 \%)$ & NS \\
\hline & 1 & & $5(1.4 \%)$ & $0(0 \%)$ & \\
\hline \multirow[t]{3}{*}{ AJCC VIII } & I & & 357 (96.5\%) & $16(94.1 \%)$ & NS \\
\hline & $\|$ & & $10(2.7 \%)$ & $1(5.9 \%)$ & \\
\hline & III & & $3(0.8 \%)$ & $0(0 \%)$ & \\
\hline \multirow[t]{3}{*}{ ATA risk of recurrence } & Low & & $171(46.2 \%)$ & $9(52.9 \%)$ & NS \\
\hline & Intermediate & & $178(48.1 \%)$ & $8(47.1 \%)$ & \\
\hline & High & & $21(5.7 \%)$ & $0(0 \%)$ & \\
\hline \multirow[t]{2}{*}{ RAl administration } & Yes & & $233(63 \%)$ & $5(30 \%)$ & 0.017 \\
\hline & Dose (mCi) & & $100(30-200)$ & $100(30-150)$ & NS \\
\hline
\end{tabular}

C-PTC: classic variant papillary thyroid cancer; WL-PTC: Warthin-like papillary thyroid cancer; $T$ T: total thyroidectomy: T+LND: total thyroidectomy + lymph node dissection; ETE: extrathyroidal extension; TgAb: Anti thyroglobulin antibodies; RAl: radioactive iodine.

Table 2. Response to treatment in the whole cohort

\begin{tabular}{|c|c|c|c|c|}
\hline & & C-PTC $(n=370)$ & WL-PTC $(n=17)$ & P-value \\
\hline \multirow[t]{2}{*}{ Structural or biochemical recurrence } & No & 331 (89.5\%) & $17(100 \%)$ & NS \\
\hline & Yes & 39 (10.5\%) & $0(0 \%)$ & NS \\
\hline \multirow[t]{5}{*}{ Response to treatment } & Excellent & $202(54 \%)$ & $4(23 \%)$ & 0.01 \\
\hline & Indeterminate by $\operatorname{Tg} 0 \operatorname{Tg} A b$ & $100(27 \%)$ & $11(65 \%)$ & 0.01 \\
\hline & Indeterminate by neck US & $29(8 \%)$ & $2(12 \%)$ & NS \\
\hline & Biochemical Incomplete & $14(4 \%)$ & $0(0 \%)$ & NS \\
\hline & Structural Incomplete & $25(7 \%)$ & $0(0 \%)$ & NS \\
\hline
\end{tabular}

C-PTC: classic variant papillary thyroid cancer; WL-PTC: Warthin-like papillary thyroid cancer; Tg: thyroglobulin; TgAb: anti thyroglobulin antibodies; US: ultrasound. 
Table 3. Response to treatment in the negative anti-thyroglobulin antibodies subgroup

\begin{tabular}{|c|c|c|c|c|}
\hline & & C-PTC & WL-PTC & P-value \\
\hline \multirow[t]{5}{*}{$\operatorname{TgAb}(-)$ subgroup } & Excellent & $150(67 \%)$ & $3(50 \%)$ & NS \\
\hline & Indeterminate by $\operatorname{Tg} 0 \mathrm{TgAb}$ & 39 (18\%) & $2(33 \%)$ & NS \\
\hline & Indeterminate by neck US & $14(6 \%)$ & $1(17 \%)$ & NS \\
\hline & Biochemical Incomplete & $6(3 \%)$ & $0(0 \%)$ & NS \\
\hline & Structural Incomplete & $13(6 \%)$ & $0(0 \%)$ & NS \\
\hline
\end{tabular}

C-PTC: classic variant papillary thyroid cancer; WL-PTC: Warthin-like papillary thyroid cancer; Tg: thyroglobulin; TgAb: anti thyroglobulin antibodies; US: ultrasound.

\section{DISCUSSION}

In this study, we compared the clinical presentation and prognosis between WL-PTC and C-PTC and found no differences in the initial risk of recurrence, AJCC staging, as well as biochemical or structural recurrence, after a median follow-up of 4.0 years.

Warthin-like PTC has been a challenging entity since its first description by Apel and cols. in 1995 (3). Its diagnosis requires an experienced pathologist, because WL-PTC can be mistaken with other oncocyterich thyroid neoplasms and lesions, such as the tall cell variant of PTC, Hürthle cell carcinoma and lymphocytic thyroiditis (6). Moreover, since the publication of the fourth edition of the WHO Classification of Tumors of Endocrine Organs, WL-PTC should also be distinguished from the oncocytic variant of PTC (5).

With respect to clinical presentation, in our cohort we found no differences between WL-PTC and C-PTC. Like other WL-PTC series, most patients were middleaged women with early-stage intrathyroidal disease and a low frequency of lymphovascular invasion and lymph node metastases. These findings are consistent with previous reports by Yeo and cols. and Erşen and cols., who described similar characteristics between both variants in diverse ethnicities $(4,6)$.

The most important finding of this study was that WLPTC and C-PTC had similar rates of both biochemical and structural recurrence. Regarding RAI ablation, it was less frequent in WL-PTC patients, which could be due to the fact that WL-PTC is a recently described variant, and by the fact that many scientific societies currently promote a more individualized management of PTC, endorsing selective use of RAI in an increasing subgroup of patients (7). For instance, $83 \%$ of our WL-PTC patients diagnosed since 2015, a period during which we demonstrated a significantly lower use of RAI. It may be argued that the more frequent use of RAI in C-PTC could have had an impact on its prognosis. However, when considering patients treated since 2015, during which RAI administration was similar between both variants, the rates of recurrence remained non-significantly different.

Regarding response to treatment, WL-PTC had a lower rate of excellent response than C-PTC. According to ATA 2015 guidelines definition, a negative neck US, negative $\mathrm{TgAb}$ and a $\mathrm{Tg}$ concentration lower than 0.2 $\mathrm{ng} / \mathrm{dL}$ are required to achieve an excellent response (7). As previously reported, we found a higher frequency of both of positive $\mathrm{TgAb}$ and lymphocytic thyroiditis in WL-PTC compared with C-PTC, which is not surprising considering that since its first description, WL-PTC has been consistently associated with chronic lymphocytic thyroiditis $(3,13)$. Considering the aforementioned conditions, the likelihood of achieving excellent response is reduced by the presence of $\mathrm{TgAb}$. However, when subgroup analysis was performed and only patients with negative $\mathrm{TgAb}$ were included, the rates of excellent response were similar in WL-PTC and C-PTC. Moreover, we have previously reported that patients with PTC and positive TgAb, in whom their concentration decrease $\geq 50 \%$ and have a negative neck US during follow-up, have a risk of recurrence comparable to patients with excellent response (10). It is noteworthy that, in our present cohort, nearly a third of $\operatorname{TgAb}$ positive patients achieved this specific, excellent-like response, most of which remained free of disease even in absence of RAI ablation.

One strength of this study is that considering that WL-PTC is an infrequent variant of PTC, to the best of our knowledge, this is the first series to report the follow-up and prognosis of patients with WL-PTC and to compare it with C-PTC $(13,14)$. We acknowledge that a median follow-up of 4 years may be deemed short and could result in the underestimation of the risk of disease recurrence. However, 317 of 387 (82\%) patients in our cohort achieved either excellent or indeterminate responses due to slightly positive $\mathrm{Tg}$ or stable/descending $\mathrm{TgAb}$, both of which have been 
previously associated with very low risks of death and structural recurrence $(7)$.

The main limitation of this study is its retrospective nature, similar to most studies in the field of PTC. However, all patients who met the inclusion criteria were followed prospectively and treated according to current ATA and local guidelines. Another limitation may be the shorter length of follow-up in the WL-PTC subgroup, owing to the relatively recent description and inclusion of this variant in thyroid cancer management care holders' practice.

We conclude that WL-PTC and C-PTC have similar clinical presentation and prognosis, so WL-PTC, although infrequent, should not be considered an aggressive variant of PTC.

Disclosure: no potential conflict of interest relevant to this article was reported.

\section{REFERENCES}

1. Davies L, Welch HG. Current thyroid cancer trends in the United States. JAMA Otolaryngol Head Neck Surg. 2014;140(4):317-22.

2. Asa SL. The Current Histologic Classification of Thyroid Cancer. Endocrinol Metab Clin North Am. 2019;48(1):1-22.

3. Apel RL, Asa SL, LiVolsi VA. Papillary Hürthle cell carcinoma with lymphocytic stroma. "Warthin-like tumor" of the thyroid. Am J Surg Pathol. 1995;19(7):810-4.

4. Erşen A, Durak MG, Canda T, Sevınç Al, Saydam S, Koçdor MA. Warthin-like papillary carcinoma of the thyroid: a case series and review of the literature. Turk Patoloji Derg. 2013;29(2):150-5.
5. Lloyd R, Osamura R, Klöppel G, Rosai J. WHO Classification of Tumours of Endocrine Organs. 4th ed. Lyon, France: IARC; 2017.

6. Yeo MK, Bae JS, Lee S, Kim MH, Lim DJ, LeeYS, et al.The WarthinLike Variant of Papillary Thyroid Carcinoma: A Comparison with Classic Type in the Patients with Coexisting Hashimoto's Thyroiditis. Int J Endocrinol. 2015;2015:456027.

7. Haugen BR, Alexander EK, Bible KC, Doherty GM, Mandel SJ, Nikiforov YE, et al. 2015 American Thyroid Association Management Guidelines for Adult Patients with Thyroid Nodules and Differentiated Thyroid Cancer: The American Thyroid Association Guidelines Task Force on Thyroid Nodules and Differentiated Thyroid Cancer. Thyroid. 2016;26(1):1-133.

8. Cooper DS, Doherty GM, Haugen BR, Hauger BR, Kloos RT, Lee $\mathrm{SL}$, et al. Revised American Thyroid Association management guidelines for patients with thyroid nodules and differentiated thyroid cancer. Thyroid. 2009;19(11):1167-214.

9. Tuttle RM, Haugen B, Perrier ND. Updated American Joint Committee on Cancer/Tumor-Node-Metastasis Staging System for Differentiated and Anaplastic Thyroid Cancer (Eighth Edition): What Changed and Why? Thyroid. 2017;27(6):751-6.

10. Zavala LF, Barra MI, Olmos R, Tuttle M, González H, Droppelmann $\mathrm{N}$, et al. In properly selected patients with differentiated thyroid cancer, antithyroglobulin antibodies decline after thyroidectomy and their sole presence should not be an indication for radioiodine ablation. Arch Endocrinol Metab. 2019;63(3):293-9.

11. Hartl DM, Hadoux J, Guerlain J, Breuskin I, Haroun F, Bidault $\mathrm{S}$, et al. Risk-oriented concept of treatment for intrathyroid papillary thyroid cancer. Best Pract Res Clin Endocrinol Metab. 2019;33(4):101281.

12. Momesso DP, Vaisman F, Yang SP, Bulzico DA, Corbo R, Vaisman $M$, et al. Dynamic Risk Stratification in Patients with Differentiated Thyroid Cancer Treated Without Radioactive lodine. J Clin Endocrinol Metab. 2016;101(7):2692-700.

13. Baloch ZW, LiVolsi VA. Warthin-like papillary carcinoma of the thyroid. Arch Pathol Lab Med. 2000;124(8):1192-5.

14. Jun HH, Kim SM, Hong SW, LeeYS, Chang HS, Park CS. Warthinlike variant of papillary thyroid carcinoma: single institution experience. ANZ J Surg. 2016;86(6):492-4. 